\title{
Research on Tracing Aircraft Assembly Error Source Based on SPC and SOFM
}

\author{
Hui Li $i^{1, a}$,Zhongqi Wang ${ }^{2, b}$,Yonggang Kang ${ }^{3, c}$ and Lan $X u^{4, d}$ \\ Key laboratory of Contemporary Design and Integrated Manufacturing Technology,Ministry of \\ Education, Northwestern Polytechnical University,Xi'an,710072,China
}

Keywords: Aircraft assembly; Trace error source; SPC; SOFM; Distance strain

\begin{abstract}
Aiming at improving the assembly quality, a method to trace the error source is proposed. Firstly, statistical process control (SPC) judges the abnormalitythrough the distribution and trend of the position errors. Then based on the measurement data from laser trackers, shape data of aircraft components is constructed to mine the small assembly data. Finally, an unsupervised learning method (SOFM) is adapted to cluster each flight to achieve error source tracing.
\end{abstract}

\section{Introduction}

Aircraft assembly is generally summarized that parts are assembled into relatively simple subassemblies,thengradually into complex components, finally into the whole aircraft[1]. Assembly deviationwhich generate,accumulates and disseminates to large components join-assembly is affected by various technological factors such as the assembly deformation, gravity, temperature, manufacturing errors, fixture deviation and measurement error etc. Such factorslead to mutual repairing and finishing. These behaviors bring the interchangeability and assembly efficiency down.Theoretically accurate analysis of error accumulation is very difficult.We form a technology strategy forassembly difficultly. Increasing the manufacturing accuracy is undesirable. Therefore, erroranalysisbased on aircraft assembly data to improveassembly qualityappears to be extremely significant[2].

Conventional aircraft assemblyerror analysistends to rely on experience of workers. Considering these, the domestic and foreign scholars have done lots of researches on error source analysis. Hu established the multi-level assembly variation propagation model and put forward SOV theory for automotive body assembly[3]. Yu used principal component analysis to isolate and identify the position variation of multi-station assembly process[4]. The basic assumptionof this method is that parts are considered as rigid body.

Aircraft join-assembly relates to cross coordination of the parts and tools. Toachieve rapid feedback of quality information in automatic join-assembly process, this paper puts forward a method of error source tracing for aircraft automatic join-assembly. This method relies on measurement data from laser trackers to mine the small assembly data.The entire error source tracingprocessis introduced in detail in Fig 1. 


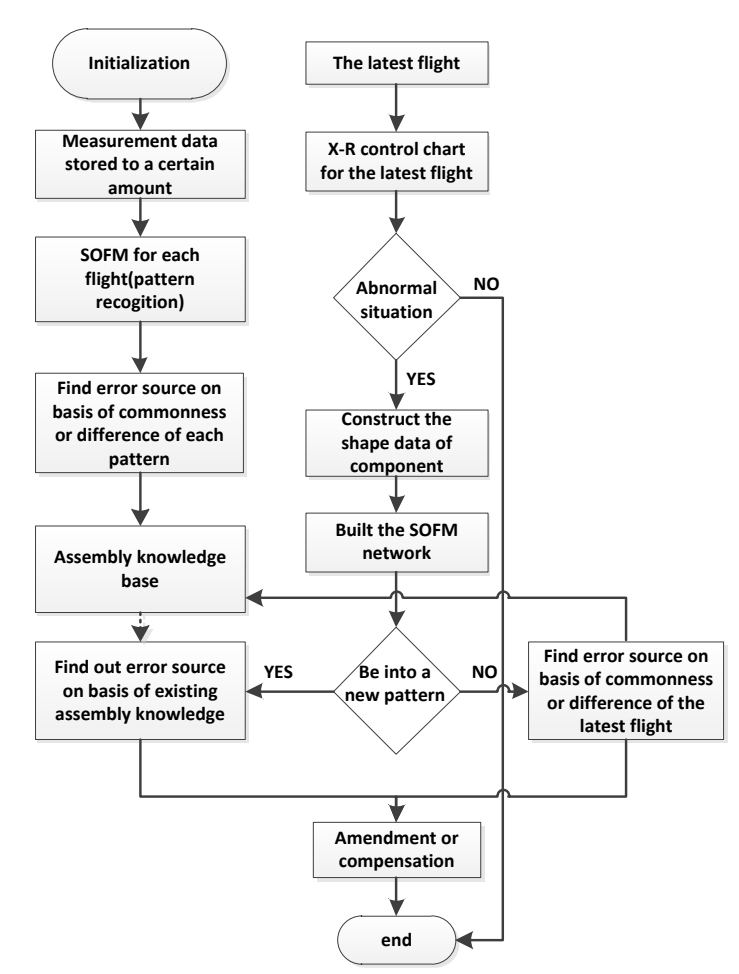

Fig .1 Theentire process of tracing error source

\section{Error Source Tracing}

\subsection{SPC to detected points}

Aircraft assemblybelongs to the category of small samples. Therefore,we use X-R control to judge abnormality rapidlyto make preparation for error source identification.

The $a$-thdetected point on aircraft components are shown in spatial position error model in Fig2.Its modulus theory position under the local coordinate system is $P_{o a}=\left[\begin{array}{ll}x_{o a} & y_{o a} z_{o a} \\ 1\end{array}\right]^{T}$. The actual position of $a$-th detected point on $i$-th flight is $P_{i a}=\left[x_{i a} y_{i a} z_{i a} 1\right]^{T}$. Transition matrix of component coordinate system to measurement coordinate system is $u_{i}$.

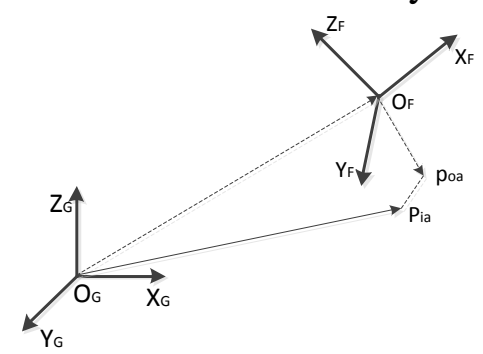

Fig 2.Spatial position error model

Let the position error be $\varepsilon_{i a}=\left[\varepsilon_{i a}^{x} \varepsilon_{i a}^{y} \varepsilon_{i a}^{z} 0\right]^{T}$,therefore $\varepsilon_{i a}=P_{i a}-U_{i} P_{o a}$.

For position error $\varepsilon_{i a}$,

$$
R_{\text {mia }}=\left|\varepsilon_{i a}-\varepsilon_{(i-1) a}\right|(1)
$$

Then

$$
\bar{R} \frac{1}{k(n-1)} \sum_{i=2}^{n} \sum_{a=1}^{k} R_{\text {mia }}(2)
$$

We know there are similar detected points on the same or different components. During the assembly,man, machine, material, method, measurementand environment are consistent. So we assume that similar detected points have the same volatility and they accord with normal distribution. According to the above, we conclude : 
For X control chart:

$$
\begin{gathered}
E\left(R_{m}\right)=\frac{2 \sigma}{\sqrt{\pi}}(3) \\
\sigma_{R_{m}}=\sqrt{2-\frac{4}{\pi} \sigma(4)}
\end{gathered}
$$

$$
\left\{\begin{array}{l}
U C L_{\varepsilon}=\stackrel{-}{\varepsilon}+3 \sigma=\bar{\varepsilon}+2.66 R_{m}^{-} \\
C L_{\varepsilon}=\bar{\varepsilon} \\
U C L_{\varepsilon}=-\bar{\varepsilon}+3 \sigma=-\bar{\varepsilon}-2.66 R_{m}^{-}
\end{array}\right.
$$

For R control chart:

$$
\left\{\begin{array}{l}
U C L_{R_{m}}=\bar{R}_{m}^{-}+3 \sigma_{R_{m}}=3.27 \bar{R}_{m}^{-} \\
C L_{R_{m}}=R_{m}^{-} \\
L C L_{R_{m}}=\bar{R}_{m}-3 \sigma_{R_{m}}=-1.27 R_{m}^{-}
\end{array}\right.
$$

$\mathrm{X}-\mathrm{R}$ control chart for aircraft detected point is shown in Fig3.Each line describes the error of the same detected point on different flight. UCL and LCL, respectively, represents top and bottom control line.CL represents control center line, that is, average.

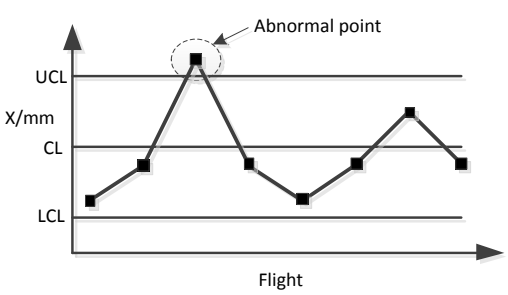

(a) X control chart

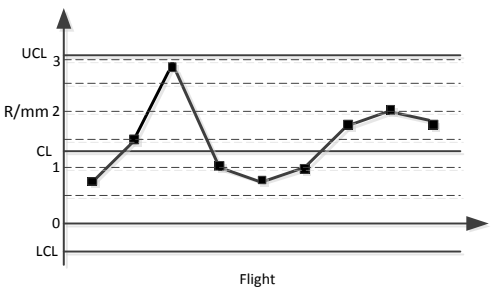

(b) R control chart

Fig.3 X-R control chart

Based on SPC todetected points, we obtain detected points stateand spot abnormal points timely.

\subsection{Pattern recognition for error source}

We diagnose detected points rapidly through SPC. When there is unusual situation, we need to trace the error source quickly. In terms of aircraft manufacturing process, priori knowledge tends to be not enough, and error is often the focus of several causes. So the efficiency of classical error diagnosis method is not high. This paper adaptspattern recognition to trace error source efficiently. However, aircraft generally belongs to small batch production.We need some time to accumulate measurement data. In addition, aircraft assembly involves lots of processes. Hence, unsupervised learning method is applied in the early stage. We obtain the estimation of measurement data. When data is stored to a certain amount, supervised learning method is applied to trace error source rapidly on basis of the previous data.

The steps of unsupervised pattern recognition are as follows: Firstly pre-treat the measurement data, then SOFM is applied toclassify all flights in a certain classification decision.

Let the actual position of the $a$-th detected point on $i$-thflight be $P_{i a}$, then $P_{i a}=U_{i} P_{o a}+V_{i a}+\Delta_{i a}$ Where:

$P_{o a}$ the theory position of the $a$-th detected point;

$U_{i}$ transition matrix of component coordinate system to the measurement coordinate system;

$V_{i a}$ system error of the $a$-th detected point on $i$-th flight, it includes manufacturing system error and assembly system error;

$\Delta_{i a}$ random error, it mainly refers to measurement error and it followsnormal distribution. 
Because system error which changes component shape does not tend to be isolated, we regard all the detected point data as shape data in order to enhance the capability of checking system error out.

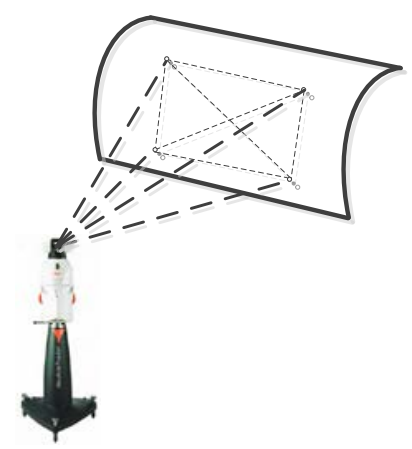

Fig.4 The shape data of component

We unit the distance data and regard it as a whole shape data to recognize changes in component shape, as shown in Fig4. The distance between $a$-thpoint and $b$-thpoint :

$$
\left|L_{i a b}\right|=\left|P_{i b}-P_{i a}\right|=\left|U_{i} L_{o a b}+\left(V_{i b}-V_{i a}\right)+\left(\Delta_{i b}-\Delta_{i a}\right)\right|(7)
$$

In order to eliminate the influence of different dimensionless distance between detected points, we introduce distance strain:

Then each component's shape is:

$$
\tau_{i a b}=\frac{\left|L_{i a b}\right|-\left|L_{o a b}\right|}{\left|L_{o a b}\right|}
$$

Next, we will take distance strain as the input data of pattern recognition.

$$
\tau_{i}=\left[\tau_{i 12}, \tau_{i 13}, \ldots, \tau_{i(k-1) k}\right]
$$

SOFM is a typical neural net which reduces the data dimensionality. SOFM doesn't require the predefined categories but classifiesthrough repeated learning. It consists of input layer and competitive layer.

Let $X=\left[x_{1}, x_{2}, \ldots, x_{n}\right]^{T} \in x$ be the input vector. It is assumed to be connected in parallel to every node in the output map. The weight vector of the nodeiis denoted by $W_{i}=\left[w_{i 1}, w_{i 2}, \ldots, w_{i n}\right]^{T} \in$ $\mathcal{R}^{n}$.The learning model is given as following:

$$
\frac{d w_{i j}(t)}{d t}=\alpha(t)\left\{\eta_{j}(t) \cdot x_{i}(t)-v\left[\eta_{j}(t)\right] \cdot w_{i j}(t)\right\}
$$

Where $\alpha(t)$ is the learning rate of the network, $v$ is the node contact frequency, $\eta_{j}$ is the neighborhood function; $w_{i j}(t)$ is the connecting weight between the output neural node $j$ and input neural node $i$.

Suppose that for the neighborhood $N E_{j}(t)$ of the node $j$, we have $\eta_{j}(t)=1$; otherwise we have $\eta_{j}(t)=0$.

Then the discrete form is[5]

$$
w_{i k}(k+1)= \begin{cases}w_{i j}(k)+\alpha(k)\left(x_{i}(k)-w_{i j}(k)\right) & j \in N E_{j}(t), \\ w_{i j}(k) & j \notin N E_{j}(t),\end{cases}
$$

This is the unsupervised learning process.The winner code is defined as the node whose weight vector has the smallest Euclidean distance.

$$
\left\|x-w_{j}(k)\right\|=\min _{i}\{\|x-w(k)\|\}
$$

As we know, NNToolbox in MATLAB provides rich functions to establish neural networks. Function newsomis used to build SOFM net to implement pattern recognition.Measurement data of different flights is clustered to identify the main error categories. When the latest fight is introduced, we cluster againif the number of measurement data increases to a certain amount. If the latest flight is classified into an existing pattern, we can speculate the error source of the latest flight according to assembly knowledge which has been formed. If not, the latest flight would come into being a new error pattern. In order to determine the specific error source of the latest flight, commonness and differences are checked out. Then the assembly knowledge is formed again. We should control the commonness or differences in the subsequent manufacturing process. If necessary, some amendment or compensation measureswould be taken to improve assembly quality. 


\section{Conclusion}

This paper proposes a method to trace error source. It overcomes the insufficiency of samples and constructs the shape data of aircraft components. It is found that tracing error source rapidlycan be realizedthrough combining SPC and SOFM.

\section{References}

[1]YunboWang. Airplane Assembly Technology [M]. Beijing: National Defense Industry Press, 1984.

[2]FengjieYu.The Small Sample Analysis of Assembly Accuracy in Aircraft Automatic Join-assembly[J]. Computer Integrated Manufacturing System, 2009.15(4).

[3]HU S J. Stream of variation theory for automotive body assembly[J].Annals of the Crip, 1997,46(1):1-6

[4]Yu Ding, DariuszCeglarek, Jianjun Shi. Dimensional Fault Diagnosis of Multistage Manufacturing Process by Using State Space Approach[J].Journal of Manufacturing Science and Engineering,2002,124:313-322.

[5]JianqiuXue.Auto Classification of Stellar Spectra Using the SOFM Method.Chinese Astronomy and Astrophysics ,2001,25:120-131. 